\title{
A Graph Signal Processing Approach to Study High Density EEG Signals in Patients with Disorders of Consciousness
}

\author{
Sepehr Mortaheb ${ }^{1,2, *}$, Jitka Annen ${ }^{1,2}$, Camille Chatelle ${ }^{1,2,3}$, Helena Cassol ${ }^{1,2}$, \\ Geraldine Martens $^{1,2}$, Aurore Thibaut ${ }^{1,2,4}$, Olivia Gosseries ${ }^{1,2}$, and Steven Laureys ${ }^{1,2}$
}

\begin{abstract}
Graph signal processing (GSP) is a novel approach to analyse multi-dimensional neuroimaging data, constraining functional measures by structural characteristics in a single framework (i.e. graph signals). In this approach, functional time series are assigned to the vertices of the underlying weighted graph and GSP analysis is performed in each time point of the signal. Here we used GSP to study local brain connectivity changes in patients with disorders of consciousness based on resting state high density electroencephalography (hdEEG) recordings. Total variation of the graph signals is a measure of signal smoothness over the underlying graph. In this study, we constructed the underlying graph based on the geometrical distances between each electrode pairs in such a way that local smoothness of the signal can be studied. Total variation analysis in $\alpha$-band showed that in the pathological states of altered consciousness, local short range communication of brain regions in this frequency band is stronger than in healthy states which shows that information is segregated in local regions in patients with disorders of consciousness.
\end{abstract}

\section{INTRODUCTION}

After a severe brain injury, $10-15 \%$ of the patients enter a condition called disorders of consciousness (DOC) [1] which includes states of coma, unresponsive wakefulness syndrome (UWS) [2], and minimally conscious state (MCS) [3]. While comatose patients are characterized by complete absence of wakefulness and awareness, UWS patients show recovery of wakefulness without signs of awareness and just have reflexive movements. MCS patients show reproducible nonreflexive movements but they are unable to communicate. These patients can be further divided into MCS- and MCS+, the latter showing signs of language preservation. When patients recover the ability to communicate and/or use object functionally, they are considered as having emerged from the MCS (EMCS) [4]. The gold standard to diagnose these patients is based on repeated behavioral assessments such as the Coma Recovery Scale-Revised (CRS-R) [5] in which different aspects of auditory, visual, motor, oromotor/verbal functions, communication, and arousal are assessed. However, it has been shown that relying solely on behavioral assessments can lead to miss covert command following ability of patients [6]. As a result, different neuroimaging methods such as functional MRI, positron emission topography, and

\footnotetext{
${ }^{1}$ GIGA-Consciousness, University of Liege, Liege, Belgium

${ }^{2}$ Coma Science Group, Neurology Department, University Hospital of Liege, Liege, Belgium

${ }^{3}$ Laboratory for NeuroImaging of Coma and Consciousness, Massachusetts General Hospital, Boston, MA, USA.

${ }^{4}$ Neuromodulation Center, Spaulding Rehabilitation Hospital, Harvard Medical School, USA

*Email: s.mortaheb@uliege.be
}

electroencephalography (EEG) have been used to study these disorders in more depth. Among them, EEG signals can be easily recorded, have high temporal resolution, and can be recorded at the bedside which is very important in this cohort of patients. High density EEG signals have been used so far to study power spectral changes, connectivity alteration between different brain regions [7], complexity analysis [8], and effect of external stimulus on the brain in DOC patients [9]. In this paper, we used the recently proposed framework of graph signal processing (GSP) [10] to analyze high density EEG signals of DOC patients constrained to the structural network derived from geometrical distances between each electrode pairs for studying information sharing characteristics and local connectivity changes of patients brain in resting state.

GSP is a framework to study signals constrained to the complex and irregular structure, defined by weighted graphs (directed or undirected) [11]. Different researchers have used various neuroimaging modalities to construct the underlying graph and the desired signal to make a graph signal for analysis. To construct the underlying weighted graph, structural connectivity can be based on diffusion weighted images [12], [13], functional connectivity between EEG electrode pairs [14], functional connectivity between brain parcellated regions based on the fMRI recordings [15], geometrical structure of the brain based on the distances between brain regions [16], or even a combination of functional and structural connectivity measures [16]. The proper choice for the underlying graph depends on the research question that one wants to answer.

In this study, we constructed a weighted graph based on the geometrical distances between electrode pairs of the high density EEG electrodes in a way that edges of the near electrodes have higher weights than the edges between far electrodes. By analyzing EEG time series constrained to this geometrical graph, a measure of information segregation in local regions of the scalp can be calculated. In section II, the mathematical background and data analysis pipeline is discussed and in section III, analysis results and their interpretation based on the designed weighted graph is stated.

\section{Materials and Methods}

\section{A. Subjects and Behavioral Assessment}

In this study, 151 patients (36 UWS, 27 MCS-, 65 MCS+, 23 EMCS) and 30 age-matched healthy subjects without any pathological neurophysiological history were included. However, subjects with high amount of signal artifact or 
TABLE I: Demographic Data of Subjects

\begin{tabular}{|c|c|c|c|c|c|c|}
\hline Group & Number & Age (years) & Gender & CRS-R Total Score & Etiology* $^{*}$ Time Since Injury (days) \\
\hline \hline UWS & 23 & $45 \pm 14.04$ & 13 Males & $5-9($ Median $=6)$ & $\begin{array}{c}\text { Anoxia }(\mathrm{n}=14) \\
\text { TBI }(\mathrm{n}=4) \\
\text { Vascular }(\mathrm{n}=5)\end{array}$ & $303 \pm 1233.3$ \\
MCS- & 19 & $41 \pm 16.29$ & 11 Males & $7-13($ Median $=9)$ & $\begin{array}{c}\text { Anoxia }(\mathrm{n}=3) \\
\text { TBI }(\mathrm{n}=9) \\
\text { Vascular }(\mathrm{n}=4) \\
\text { Mixed }(\mathrm{n}=3)\end{array}$ & $465 \pm 922.15$ \\
\hline MCS+ & 42 & $31 \pm 18.24$ & 27 Males & $7-23($ Median $=13)$ & $\begin{array}{c}\text { Anoxia }(\mathrm{n}=8) \\
\text { TBI }(\mathrm{n}=25) \\
\text { Vascular }(\mathrm{n}=8) \\
\text { Mixed }(\mathrm{n}=1)\end{array}$ & $661.5 \pm 1218.8$ \\
\hline EMCS & 12 & $32 \pm 15.24$ & 9 Male & $15-23($ Median $=21.5)$ & $\begin{array}{c}\text { Anoxia }(\mathrm{n}=2) \\
\text { TBI }(\mathrm{n}=7) \\
\text { Vascular }(\mathrm{n}=3)\end{array}$ & $418 \pm 1850.7$ \\
\hline Healthy & 28 & $42.5 \pm 14.53$ & 14 Male & - & - & - \\
\hline
\end{tabular}

*TBI: Traumatic Brain Injury, Mixed: TBI + Anoxia

less than 5 minutes of clean signal after preprocessing were removed from further analysis. At the end, 124 subjects (23 UWS, 19 MCS-, 42 MCS+, 12 EMCS, 28 Healthy) were remained for analysis. The demographic data of all analyzed subjects are reported in table I. Repeated CRS-R assessments by trained clinicians were used for clinical diagnosis of the patients (minimum five assessments), and the final diagnosis was based on the best diagnosis obtained across the assessments. All the data were recorded at the University Hospital of Liege, Belgium, and informed consents were signed by subjects or their legal representatives and the study was approved by the local ethics committee.

\section{B. EEG Signal Recording and Preprocessing}

High density EEG data were recorded using 256 electrode EGI setup with $250 \mathrm{~Hz}$ sampling rate (or $500 \mathrm{~Hz}$ which further downsampled to $250 \mathrm{~Hz}$ ) in resting state condition with eyes open for at least 20 minutes. Data were first bandpass filtered (1 to $30 \mathrm{~Hz}$ ) and bad channels were rejected by visual inspection. Further, signals were segmented into 2 seconds epochs. Epochs with amplitude higher than 100 $\mu V$ were rejected automatically and after this initial cleaning the remaining bad epochs were rejected by visual inspection. Eye blinks, muscle, and ECG artifacts were removed from the data using independent component analysis. Neural and non-neural components which contribute in $99 \%$ of the signal variance were checked by visual inspection and non-neural components were set to zero and clean signal were reconstructed by remaining neural components. Finally, bad channels were interpolated using spherical interpolation and data were re-referenced to the common average of all electrodes. The first 150 epochs of each subjects (i.e. 5 minutes) were used for further analysis.

\section{Graph Signal Processing}

Here, we limit our discussion to undirected graphs. Suppose that we have an undirected graph $G=\{V, E, \mathbf{W}\}$ where $V$ is the finite vertices set with $|V|=N, E$ is the edges set, and $\mathbf{W}$ is the weighting matrix, representing the weights of the edges. Every edge between nodes $i$ and $j$ has a weight $w_{i j}$ and $w_{i j}=0$ shows absence of connection between the two nodes. If we assign each sample of the signal $\mathbf{s}=\left[s_{0}, s_{1}, \ldots, s_{N}{ }_{1}\right]^{T}$ to a specific node of the graph $G$ we would have a graph signal to analyze. Based on this definition, the derivative of the signal with respect to the edge $e=(i, j)$ can be defined as:

$$
\frac{\partial \mathbf{s}}{\partial e_{i, j}} \triangleq \sqrt{w_{i j}}\left|s_{j}-s_{i}\right| \text {. }
$$

As a result, the gradient of signal $\mathbf{s}$ on the vertex $i$ is defined as an $\mathrm{N}$ dimensional vector:

$$
\Delta_{i} \mathbf{s} \triangleq\left[\frac{\partial \mathbf{s}}{\partial e_{i, j}}\right] \text { for } j=0,1, \ldots, N-1 .
$$

$L 2$-norm of the gradient of the signal over node $i$ is a measure of local variation and local smoothness of the signal s over that node:

$$
\left\|\Delta_{i} \mathbf{S}\right\|_{2}=\left[\sum_{j=0}^{N} w_{i j}\left(s_{j}-s_{i}\right)^{2}\right]^{\frac{1}{2}} .
$$

Based on this definition, total variation (TV) of the signal over the entire graph can be introduced as the sum of local variations:

$$
T V(\mathbf{s})=\sum_{i=0}^{N}\left[\sum_{j=0}^{N} w_{i j}\left(s_{j}-s_{i}\right)^{2}\right]^{\frac{1}{2}} .
$$

Total variation is a measure of smoothness of the signal over the underlying graph. Lower values of TV shows that nodes which are connected to each other with high weights have near values, while higher values of TV show that signal is not following the underlying graph structure and connected nodes have dissimilar values. This concept is useful to study connectivity and information flow of the brain regions based on different signal modalities (e.g. EEG, fMRI, and PET).

\section{Data Analysis Pipeline}

The whole pipeline of data analysis is shown in Fig.1. The underlying graph of the graph signals were constructed based on the geometrical distances between each electrode 


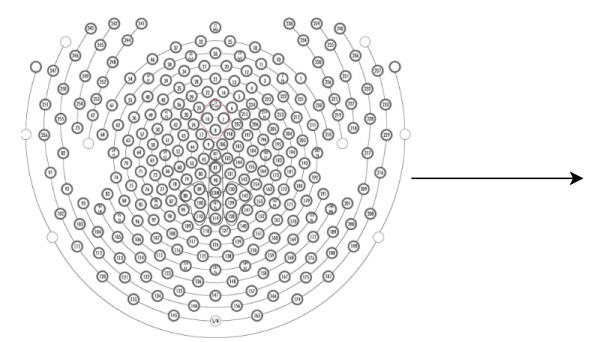

(a)

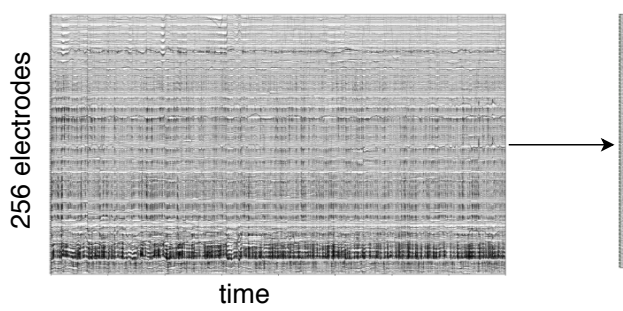

(c)

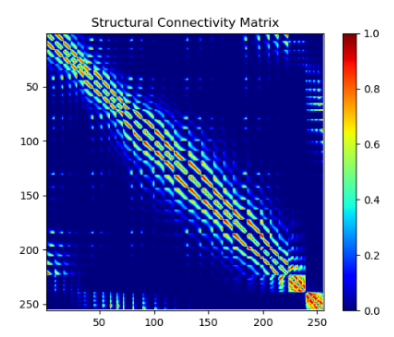

(b)

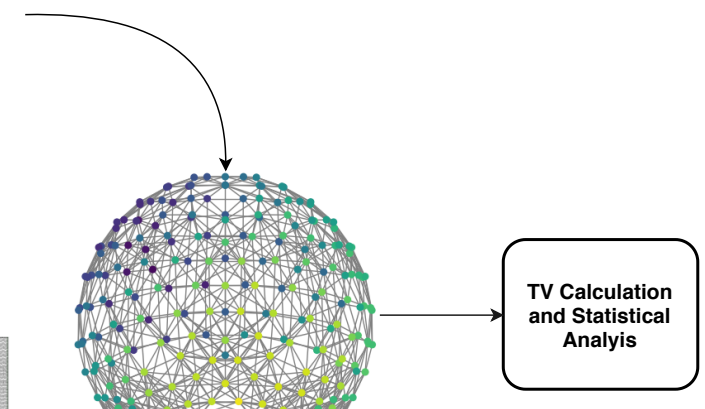

(f)

(e)

Fig. 1: Analysis pipeline of the study. (a) Scheme of the 256-electrode EGI (Geodesics) electrode configuration. (b) Underlying weighted graph, constructed based on the geometrical distances between each pair of the electrodes of EGI cap using eq.5. (c) Raw EEG signals recorded in the resting state and bandpass filtered in $[1-30]$ Hz frequency band. (d) Epoched EEG signal after preprocessing and $\alpha$ band extraction. t0 is an example signal time point to construct a graph signal. (e) A sample graph signal which is constructed based on the weighted graph and the signal extracted from each time point of the EEG recording. Just the $60 \%$ strongest edges are shown for visualization purposes. (f) Total variation is calculated for each time point graph signal of each epoch and their mean is considered as the total variation of the epoch. Then median value of the epoch total variations is considered as the subject total variation.

pairs (based on the standard EGI 256-electrode location file) using a Gaussian kernel weighting function:

$$
w_{i j}=\exp \left(-\frac{d(i, j)^{2}}{2 \rho^{2}}\right),
$$

where $d(i, j)$ is the geometrical distance between electrodes $i$ and $j$, and $\rho$ is a regularization parameter which determines the strength of connection for near and far electrode pairs. With this definition, near vertices (electrodes) are strongly connected while physically far vertices have weak connections. However, based on this formula, all the vertices are connected to each other and the underlying structural graph is a complete graph. Because the weight of the graph edges is higher for near electrodes, this graph is suitable for analysis of signal variation in local areas and short range communications. Because the weight of far electrode pairs are nearly zero in our designed weighted graph, we cannot infer any result about the long range communication of the brain regions in this framework.

Based on the previous studies, EEG $\alpha$-band $(8-12 \mathrm{~Hz})$ plays an important role in analysis of DOC patients [7]. For this reason, and the relatively low contribution of (non)physiological artifacts in this band, we focused on this frequency band in the current analysis. After signal cleaning, the $\alpha$ band was extracted from each epoch and the total variation of the graph signal of each time point was calculated (as 4). Considering sampling frequency of $250 \mathrm{~Hz}$, each epoch has 500 time points and so 500 graph signals. The TV was calculated at each time point and the mean TV of all 500 graph signals at each epoch was considered as the TV of the epoch. Finally, median of the TV of all epochs were reported as the TV of the subject.

\section{E. Statistical Analysis}

Any significant difference among groups in terms of age and time since injury was tested using one-way anova. To see the difference between TV of different groups pairs, independent t-test were performed. In addition, a Spearman correlation test was done to investigate possible relationship between TV and CRS-R total score.

\section{RESULTS AND DISCUSSION}

There were no significant difference among patients groups and healthy subjects in terms of age $(F=1.61$, $p=0.18)$. In addition, no significant difference was seen among patients groups in terms of time since injury $(F=$ $0.93, p=0.43)$. The bar plot of total variation of the subjects in each group is shown in Fig.2. As it can be seen qualitatively, there is an increase in the median value of the TV with increased level of consciousness. No significant differences were observed between UWS and MCS- and between MCS- and EMCS. However, significant differences 


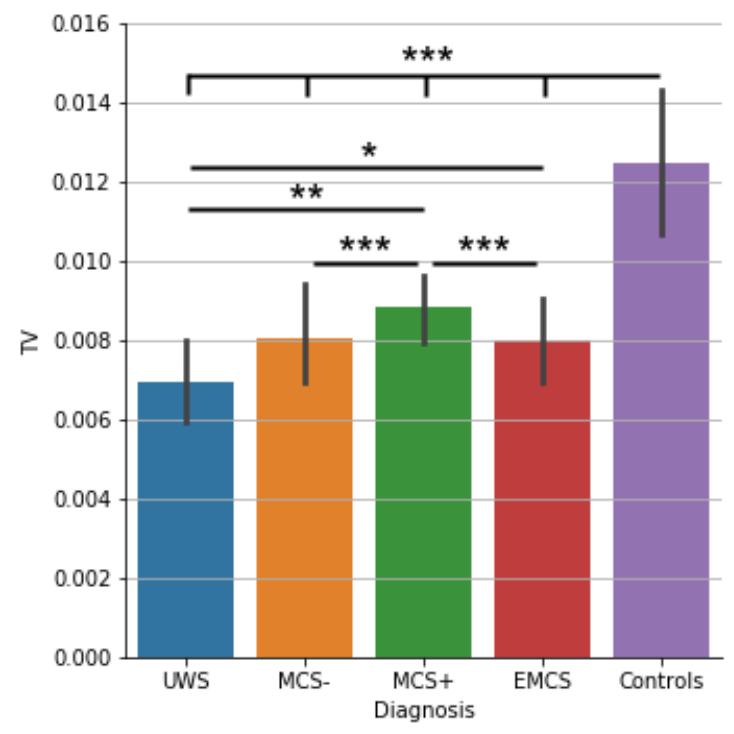

Fig. 2: Bar plot of TV values in $\alpha$-band for each group of subjects. $(*: \mathrm{p}<0.05, * *: \mathrm{p}<0.01, * * *: \mathrm{p}<0.001$, uncorrected)

were observed between any other group pairs. To study the relationship between TV of the EEG signal constrained to the underlying weighted graph and level of consciousness, we further performed a Spearman correlation analysis between TV and CRS-R total score. As it can be seen in Fig.3, there is a significant positive correlation between these two measures $\left(R^{2}=0.801, \mathrm{p}<0.001\right)$.

Based on the Eq. (4), two parameters are important in calculation of the TV: weight of the edge connecting two graph nodes to each other $\left(w_{i j}\right)$ and the squared magnitude of the difference of values assigned to the related graph nodes $\left(\left(s_{j}-s_{i}\right)^{2}\right)$. If $w_{i j} \simeq 0$, the difference term would not have important impact on the TV value and so $w_{i j}\left(s_{j}-s_{i}\right)^{2} \simeq 0$. As a result, higher values of the TV shows that $w_{i j}$ should be a strong connection and the difference term of $\left(s_{j}-s_{i}\right)$ should have a high value. In this study, the designed weighted graph is such that near electrodes have strong connection while far electrodes have very weak connections. As a result, TV value of each subject is a measure of signal similarity in neighboring electrodes. Higher values of the TV shows that signal in local areas are fast varying and signal values of the neighboring electrodes are dissimilar. Therefore, this framework is suitable for analyzing local communications of the brain. Based on our analysis of high density resting state EEG signals in $\alpha$-band, it was shown that as the level of consciousness decreases, the TV value also decreases, which shows information segregation of the $\alpha$-band network in pathological states of consciousness is higher than normal conditions. These findings are in line with the results of other studies such as [7] which showed the $\alpha$-network has strong local functional connections and weak long range communication in DOC patients based on the debiased weighted Phase-Lag Index (dwPLI) connectivity metric. In addition, previous studies on cortical stimulation

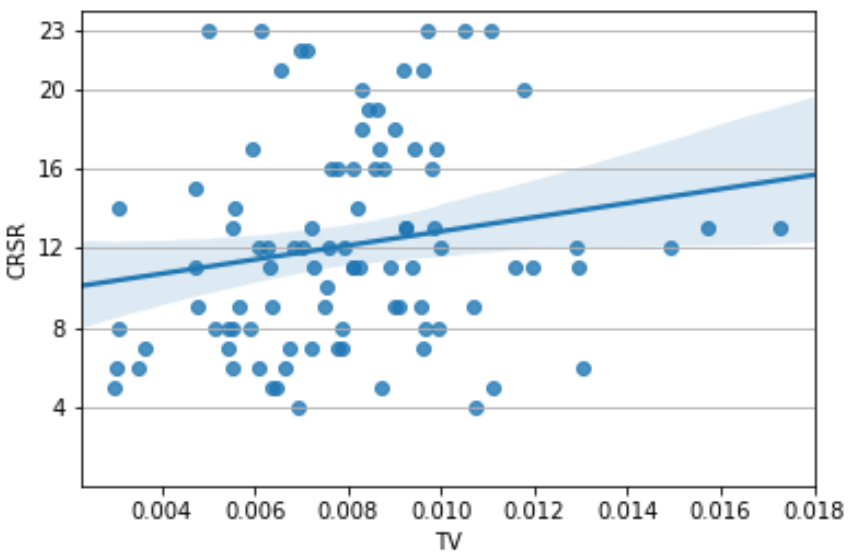

Fig. 3: Correlation between total variation (TV) and CRS-R total score shows significant linear relationship between TV and level of consciousness $\left(R^{2}=0.801, \mathrm{p}<0.001\right)$.

using transcranial magnetic stimulation combined with EEG (TMS-EEG) has shown local EEG responses to the stimulation in UWS patients which supports our findings [17], [18]. This shows the clinical advantage of our method that only uses resting state recordings for this kind of analysis and there is no need for cortical stimulation. However, because of the overlap between TV values of different groups, it is not still appropriate for single subject analysis which is not the case for TMS-EEG studies.

Future GSP analyses could consider connectivity in the temporal and spatial domain for the supporting weighted graph in different frequency bands and with higher number of patients, to provide a truly multi-modal analysis in DOC patients. This will include combination of functional or effective connectivity matrices with the geometrical distance matrix to have both directed and undirected graphs for GSP analysis. Also, future studies should consider designing a suitable graph for long range information flow analysis (information integration) and combine it with the current study to make a complete framework for GSP analysis of the signals.

\section{CONCLUSION}

In this study, a framework based on graph signal processing was designed to study local EEG connectivity changes of patients with disorders of consciousness. Total variation analysis of the graph signals of the high density EEG recordings in the $\alpha$-band showed lower signal variation constrained to the underlying structural graph in pathological states of consciousness which shows information segregation in lower levels of the consciousness.

\section{ACKNOWLEDGMENT}

The study was supported by the University and University Hospital of Liege, the Belgian National Funds for Scientific Research (FRS-FNRS), the European Unions Horizon 2020 Framework Programme for Research and Innovation 
under the Specific Grant Agreement No. 785907 (Human Brain Project SGA2), the Luminous project (EU-H2020fetopenga686764), the European Space Agency (ESA) and the Belgian Federal Science Policy Office (BELSPO) in the framework of the PRODEX Programme,the CenterTBI project (FP7-HEALTH- 602150), the Public Utility Foundation Universit Europenne du Travail, Fondazione Europea di Ricerca Biomedica, the Bial Foundation, the Mind Science Foundation, the European Commission, and Marie Sklodowska-Curie (H2020-MSCA-IF-2016-ADOC-752686).

\section{REFERENCES}

[1] H. S. Levin, C. Saydjari, H. M. Eisenberg, M. Foulkes, L. F. Marshall, R. M. Ruff, J. A. Jane, and A. Marmarou, "Vegetative state after closed-head injury: a traumatic coma data bank report," Archives of neurology, vol. 48, no. 6, pp. 580-585, 1991.

[2] S. Laureys, G. G. Celesia, F. Cohadon, J. Lavrijsen, J. León-Carrión W. G. Sannita, L. Sazbon, E. Schmutzhard, K. R. von Wild, A. Zeman, et al., "Unresponsive wakefulness syndrome: a new name for the vegetative state or apallic syndrome," BMC medicine, vol. 8 , no. 1 , p. $68,2010$.

[3] J. T. Giacino, S. Ashwal, N. Childs, R. Cranford, B. Jennett, D. I. Katz, J. P. Kelly, J. H. Rosenberg, J. Whyte, R. Zafonte, et al., "The minimally conscious state: definition and diagnostic criteria," Neurology, vol. 58, no. 3, pp. 349-353, 2002.

[4] O. Gosseries, H. Di, S. Laureys, and M. Boly, "Measuring consciousness in severely damaged brains," Annual Review of Neuroscience, vol. 37, pp. 457-478, 2014.

[5] J. T. Giacino, K. Kalmar, and J. Whyte, "The JFK coma recovery scalerevised: Measurement characteristics and diagnostic utility1," Archives of physical medicine and rehabilitation, vol. 85, no. 12, pp. 2020 2029, 2004.

[6] M. M. Monti, A. Vanhaudenhuyse, M. R. Coleman, M. Boly, J. D. Pickard, L. Tshibanda, A. M. Owen, and S. Laureys, "Willful modulation of brain activity in disorders of consciousness," New England Journal of Medicine, vol. 362, no. 7, pp. 579-589, 2010.

[7] S. Chennu, P. Finoia, E. Kamau, J. Allanson, G. B. Williams, M. M Monti, V. Noreika, A. Arnatkeviciute, A. Canales-Johnson, F. Olivares, et al., "Spectral signatures of reorganised brain networks in disorders

[15] W. Huang, L. Goldsberry, N. F. Wymbs, S. T. Grafton, D. S. Bassett, and A. Ribeiro, "Graph frequency analysis of brain signals.," J. Sel. Topics Signal Processing, vol. 10, no. 7, pp. 1189-1203, 2016. of consciousness," PLoS computational biology, vol. 10, no. 10, p. e1003887, 2014.

[8] J.-R. King, J. D. Sitt, F. Faugeras, B. Rohaut, I. El Karoui, L. Cohen, L. Naccache, and S. Dehaene, "Information sharing in the brain indexes consciousness in noncommunicative patients," Current Biology, vol. 23, no. 19, pp. 1914-1919, 2013.

[9] M. Napolitani, O. Bodart, P. Canali, F. Seregni, A. Casali, S. Laureys, M. Rosanova, M. Massimini, and O. Gosseries, "Transcranial magnetic stimulation combined with high-density eeg in altered states of consciousness," Brain injury, vol. 28, no. 9, pp. 1180-1189, 2014.

[10] D. I. Shuman, S. K. Narang, P. Frossard, A. Ortega, and P. Vandergheynst, "The emerging field of signal processing on graphs: Extending high-dimensional data analysis to networks and other irregular domains," IEEE Signal Processing Magazine, vol. 30, no. 3, pp. 8398, 2013.

[11] A. Ortega, P. Frossard, J. Kovačević, J. M. Moura, and P. Vandergheynst, "Graph signal processing: Overview, challenges, and applications," Proceedings of the IEEE, vol. 106, no. 5, pp. 808-828, 2018.

[12] W. Huang, T. A. Bolton, J. D. Medaglia, D. S. Bassett, A. Ribeiro, and D. Van De Ville, "A graph signal processing perspective on functional brain imaging," Proceedings of the IEEE, 2018.

[13] J. D. Medaglia, W. Huang, E. A. Karuza, A. Kelkar, S. L. ThompsonSchill, A. Ribeiro, and D. S. Bassett, "Functional alignment with anatomical networks is associated with cognitive flexibility," Nature Human Behaviour, vol. 2, no. 2, p. 156, 2018.

[14] K. Smith, B. Ricaud, N. Shahid, S. Rhodes, J. M. Starr, A. Ibáñez, M. A. Parra, J. Escudero, and P. Vandergheynst, "Locating temporal functional dynamics of visual short-term memory binding using graph modular dirichlet energy," Scientific reports, vol. 7, p. 42013, 2017.

[16] M. Ménoret, N. Farrugia, B. Pasdeloup, and V. Gripon, "Evaluating graph signal processing for neuroimaging through classification and dimensionality reduction," in Signal and Information Processing (GlobalSIP), 2017 IEEE Global Conference on, pp. 618-622, IEEE, 2017.

[17] M. Rosanova, O. Gosseries, S. Casarotto, M. Boly, A. G. Casali, M.-A. Bruno, M. Mariotti, P. Boveroux, G. Tononi, S. Laureys, et al., "Recovery of cortical effective connectivity and recovery of consciousness in vegetative patients," Brain, vol. 135, no. 4, pp. 13081320,2012

[18] O. Gosseries, S. Sarasso, S. Casarotto, M. Boly, C. Schnakers, M. Napolitani, M.-A. Bruno, D. Ledoux, J.-F. Tshibanda, M. Massimini, et al., "On the cerebral origin of eeg responses to tms: insights from severe cortical lesions," Brain stimulation, vol. 8, no. 1, pp. 142$149,2015$. 